\title{
El POTENCIAL PEDAGÓGICO DEL PROYECTO INTEGRADOR COMO ESTRATEGIA DE AULA: ESTUDIO DE CASO EN EL PROGRAMA DE TECNOLOGÍA INDUSTRIAL DE LA UNIVERSIDAD DE SANTANDER (UDES)
}

\author{
Claudia Jazmín Galeano-Barrera ${ }^{1}$, William Humberto Zamudio-Peña ${ }^{2}$, \\ Viviana Duro-Novoa ${ }^{3}$, Ana Francisca Martínez-Quintero ${ }^{4}$
}
1 Magíster en Desarrollo Sostenible y Medio Ambiente, Coordinadora de Tecnología Industrial/Gestión Industrial, Centro de Estudios Tecnológicos, Universidad de Santander uDEs, Bucaramanga, Colombia
2 Magister en Administración de Empresas, coordinador de Investigación, Centro de Estudios Tecnológicos, Universidad de Santander udes, Bucaramanga, Colombia. Correo electrónico: william.zamudio@udes.edu.co 3 Magíster en Gestión de la Información, profesora de la Facultad de Ciencias Económicas, Universidad de la Habana, Cuba
4 Especialista en Gestión de la Tecnología Educativa, Directora del Centro de Estudios Tecnológicos, Universidad de Santander UDES, Bucaramanga, Colombia

Fecha de recibido: 18 de octubre del $2016 \quad$ Fecha de aprobado: 30 de marzo del 2017

Cómo citar este artículo: C. J. Galeano-Barrera, W. H. Zamudio-Peña, V. Duro-Nova, A. F. Martínez-Quintero, "El potencial pedagógico del proyecto integrador como estrategia de aula; estudio de caso en el programa de tecnología industrial de la Universidad de Santander udes", Ingeniería Solidaria, vol. 13, n. ${ }^{\circ}$ 22, mayo de 2017, pp. 153-169. doi: http://dx.doi. org/10.16925/in.v13i22.1851

Resumen. Introducción: el presente artículo sintetiza las experiencias de aula obtenidas en la aplicación de "El proyecto integrador como estrategia de aula en los cursos de Tecnología Industrial”, desarrollado en cuarto semestre del programa tecnológico durante 2013 y 2014 en la Universidad de Santander (UDES). El objetivo del presente trabajo, realizado durante el 2015, se enfoca en demostrar que la apropiación e integración de los conocimientos de los estudiantes del programa se materializa en productos prototipo de innovación y en trabajos de grado enfocados en el emprendimiento. Metodología: en el desarrollo del proyecto integrador se utilizó la metodología de proyecto formativo para el desarrollo de competencias básicas, profesionales e investigativas del estudiante. La experiencia pedagógica permite un espacio participativo de los estudiantes en formación, al proponer ideas innovadoras como respuesta a la problemática de contexto, así como al aprovechar los conocimientos adquiridos y el uso de recursos de la universidad. Resultados: la apropiación del proyecto integrador por parte del estudiante estimula su creatividad para fabricar el prototipo de su propuesta. El conjunto de prototipos se dona a una comunidad rural de Bucaramanga, lo que fortalece la alianza de la investigación y la extensión, incluyendo la gestación de su trabajo de grado. Conclusiones: el fortalecimiento de competencias orientadas al saber hacer, permite al estudiante formarse de manera íntegra, lo alimenta de conocimientos, le permite hacer uso de los mismos (praxis), y lo posiciona en el mercado con capacidad emprendedora.

Palabras clave: aprendizaje, educador, emprendimiento, innovación, proyecto integrador. 


\title{
The Pedagogical Potential of the Integrative Project as a Classroom Strategy: A Case Study in the Industrial Technology Program at the Universidad de Santander (Udes)
}

\begin{abstract}
Introduction: This article summarizes the classroom experiences obtained in the application of "The integrative project as a classroom strategy in Industrial Technology courses", carried out in the fourth semester of the technological program in 2013 and 2014, at the Universidad de Santander (UDEs). The objective of this work, carried out during 2015, is to show that the appropriation and integration of the students' knowledge is materialized in prototype innovation products and in dissertations focused on entrepreneurship. Method: In the course of the integrative project, the formative project methodology was used for building the student's basic, professional and research skills. The pedagogical experience provides students with the opportunity to participate by proposing innovative ideas as a response to the context problem, as well as by taking advantage of the acquired knowledge and the use of university resources. Results: Appropriation of the integrative project by the student stimulates his creativity to produce the prototype of his proposal. The set of prototypes is donated to a rural community in Bucaramanga, which strengthens the research-outreach alliance, including the preparation of dissertations. Conclusions: The strengthening of skills oriented to the know-how allows the student to be trained comprehensively, feeds him with knowledge, allows him to use it (praxis), and positions him in the market with entrepreneurial ability.
\end{abstract}

Keywords: learning, educator, entrepreneurship, innovation, integrative project.

\section{O POTENCIAL PEDAGÓgICO DO PROJETO INTEGRADOR COMO}

\section{ESTRATÉgia de SALA DE AULA: ESTUdo DE CASO NO PROGRAMA DE TeCNOlogia INdustrial da UNiversidad DE SANTANDER (Udes)}

Resumo. Introdução: este artigo sintetiza as experiências de aula obtidas na aplicação de "O projeto integrador como estratégia de sala de aula nos cursos de tecnologia industrial", desenvolvido no quarto período do programa técnico, em 2013 e 2014, na Universidad de Santander (Udes). O objetivo deste trabalho, realizado durante 2015, é demonstrar que a apropriação e a integração dos conhecimentos dos estudantes do programa se materializa em produtos protótipo de inovação e em trabalhos de conclusão de curso focados no empreendimento. Metodologia: no desenvolvimento do projeto integrador, utilizou-se a metodologia de projeto formativo para o desenvolvimento de competências básicas, profissionais e investigativas do estudante. A experiência pedagógica permite um espaço participativo dos estudantes em formação ao propor ideias inovadoras como resposta à problemática de contexto, bem como ao aproveitar os conhecimentos adquiridos e o uso de recursos da universidade. Resultados: a apropriação do projeto integrador por parte do estudante estimula sua criatividade para fabricar o protótipo de sua proposta. O conjunto de protótipos é doado a uma comunidade rural de Bucaramanga (Colômbia), o que fortalece a parceria da pesquisa e da extensão, incluindo a elaboração de seu trabalho de conclusão de curso. Conclusões: o fortalecimento de competências orientadas ao saber fazer permite ao estudante formar-se de maneira íntegra, alimenta-o de conhecimentos, permite que faça uso deles (práxis) e posiciona-o no mercado com capacidade empreendedora.

Palavras-chave: aprendizagem, educador, empreendimento, inovação, projeto integrador. 


\section{Introducción}

Según Etzkowitz y Leydesdorff, el modelo universidad, gobierno y empresa busca aprovechar las dinámicas universitarias como gestor de conocimiento, de manera que contribuye a las relaciones entre el Estado y la empresa, y los resultados de la interacción entre las tres partes para el impulso de la innovación empresarial como apalancamiento del desarrollo social, económico y sostenible de la sociedad [1].

Como plantean Fernández, Castro, Consea y Gutiérrez [2], la institucionalización de las relaciones de las universidades con el entorno socioeconómico es más fructífera cuando las universidades se han ganado un prestigio en su ámbito geográfico de influencia, ya sea por la cualificación de los profesionales que forma, o bien gracias a la capacidad demostrada por los miembros de la comunidad académica para resolver las demandas planteadas por los elementos de dicho entorno en los ámbitos científico-técnicos. A partir del momento en el que las universidades se han ganado el respeto de la sociedad que las financia es que cabe plantearse la generación de una corriente de opinión entre los miembros de sus comunidades universitarias que sirva para dinamizarlos hacia actividades de I+D y de transferencia de conocimientos al entorno socioeconómico.

La Universidad deSantander, en Bucaramanga, Colombia, entre sus objetivos establece la generación, transferencia y aplicación del conocimiento científico y tecnológico en todas sus formas y expresiones a los sectores económicos y sociales, con el fin de contribuir a la solución de las necesidades y problemas de la región y del país.

De igual manera, en el Plan de Desarrollo Institucional UDES 2013-2018 [3] se contempla el empresarismo como un factor que cobra cada vez más importancia en las políticas de apoyo a la competitividad, asociado al fomento de una cultura del emprendimiento entre los habitantes de un territorio, en la medida en que este constituye una alternativa para promover la innovación y la estabilidad económica de sus actores, al tiempo que contribuye a generar valor agregado y empleo en un territorio, por medio de la creación de empresas.

Mediante la interdisciplinariedad, la investigación y los problemas de contexto, el proyecto integrador propone generar desde el aula de clase, soluciones innovadoras para la formulación y desarrollo de alternativas de solución, direccionadas a las problemáticas del entorno, lo cual permite la apropiación e integración de los conocimientos de los estudiantes del programa, direccionada hacia la innovación y el emprendimiento.

\subsection{Naturaleza del proyecto formativo}

Desde la interdisciplinariedad, la investigación y los problemas de contexto, el proyecto tiene un énfasis en la aplicación tecnológica por la generación de soluciones innovadoras desde el aula para la formulación y desarrollo de proyectos integradores, direccionados a resolver las problemáticas del entorno.

\subsection{Problema del contexto que propende a abordar el proyecto formativo}

Se plantea con relación al problema de contexto el siguiente interrogante: ¿cómo desarrollar desde el aula, la apropiación e integración de los conocimientos adquiridos por los estudiantes del programa de Tecnología Industrial, para el fomento de las habilidades propias de la innovación y el emprendimiento?

\subsection{Competencias que se desean formar}

De acuerdo con los lineamientos consignados en el Proyecto Educativo Institucional udes [4], en su estructura curricular se plantea el desarrollo de competencias básicas, específicas, genéricas, profesionales e investigativas. A lo cual, el programa de tecnología industrial, desde la aplicación de proyectos integradores, da cumplimiento (véase la tabla 1). 
Tabla 1. Competencias de los proyectos integradores

\begin{tabular}{|c|c|}
\hline Básicas y genéricas & $\begin{array}{l}\text { - Genera habilidades comunicativas, orales y escritas para una mejor interrelación con su } \\
\text { entorno. } \\
\text { - Integra el conocimiento cuantitativo sobre aspectos espaciales de la realidad para analizar } \\
\text { y resolver problemas relacionados con la vida cotidiana y con el mundo laboral. } \\
\text { - Conoce y aplica con juicio crítico y objetivo los medios tecnológicos e informáticos que } \\
\text { le permitan acceder a distintos ambientes de información, conocimiento y pensamiento. } \\
\text { - Capacidad para la interdisciplinariedad y el trabajo en equipo, al propiciar la solución } \\
\text { efectiva a cada una de las necesidades presentes en el entorno. }\end{array}$ \\
\hline $\begin{array}{l}\text { Profesionales y } \\
\text { específicas }\end{array}$ & $\begin{array}{l}\text { - Capacidad de observación, análisis y síntesis, en relación con un pensamiento sistémico. } \\
\text { - Determina el tiempo estándar y el mejor método para la fabricación de un producto. } \\
\text { - Identifica el cuello de botella en la producción y realiza el diagrama hombre- máquina. } \\
\text { - Identifica los posibles riesgos existentes en la producción para presentar el plan de miti- } \\
\text { gación o eliminación. } \\
\text { - Desarrolla la matriz de riesgos derivada de la identificación de factores de riesgos en la } \\
\text { - } \text { producción. } \\
\text { - Recontifica los elementos de protección personal para mitigar los riesgos. } \\
\text { - Diseña el empaque y etiqueta del producto a fabricar. } \\
\text { - Determina la cadena de abastecimiento y logística inversa, dentro de la organización. }\end{array}$ \\
\hline Investigativas & $\begin{array}{l}\text { - Apropia y adapta los conocimientos científicos con el fin de generar soluciones a proble- } \\
\text { máticas empresariales. } \\
\text { - Formula proyectos de aplicación de sus conocimientos para ofrecer alternativas de solu- } \\
\text { ción a un problema detectado según el objeto de estudio. } \\
\text { - Desarrollo de habilidades, destrezas, valores y actitudes hacia el emprendimiento, la in- } \\
\text { novación y nuevos modelos empresariales. }\end{array}$ \\
\hline
\end{tabular}

Fuente: elaboración propia

\section{Estado de arte del proyecto integrador como experiencia pedagógica}

Un proyecto integrador se incorpora a la educación como una estrategia curricular que permite generar una nueva vía para que los estudiantes desarrollen competencias, lo que significa el deber de los estudiantes por contemplar oportunidades para aprender a actuar de forma integral y no individualizada. Todo proyecto busca abordar problemas en el contexto, y en ese sentido, es la estrategia más integral para la formación y evaluación de las competencias [5].

Tiene como propósito desarrollar una educación de calidad superior tecnológica, así como favorecer una formación profesional integral que privilegie la percepción analítica y crítica de los fenómenos de la globalización, y del cambio de criterios y estándares en los productos, con el fin de responder a las actuales condiciones que generan y determinan nuevos y más estrictos mecanismos de competitividad nacional e internacional. El proyecto integrador, más que una estrategia didáctica que consiste en realizar un conjunto de actividades articuladas entre sí, con un inicio, un desarrollo y un final cuyo propósito es identificar, interpretar, argumentar y resolver un problema del contexto, y así contribuir a formar una o varias competencias del perfil de egreso de acuerdo con el abordaje de un problema significativo del contexto disciplinar-investigativo, social o laboral-profesional, etc. [6], es una estrategia metodológica y evaluativa de investigación, direccionada al planteamiento y solución de problemas relacionados con la práctica profesional y la calidad de vida. No obstante, requiere de la articulación de asignaturas del nivel, disciplina o carrera.

La metodología de los proyectos formativos fue propuesta por Tobón a finales de los años noventa con base en las contribuciones originales de Kilpatrick 
(1918). Esto se hizo integrando las competencias, el proyecto ético de vida y los procesos de emprendimiento creativo a partir de proyectos de investigación en el aula con docentes de todos los niveles educativos en diferentes países de Iberoamérica $[5,7,8]$.

Uno de los ejemplos de proyectos integrados de semestre como estrategia de aprendizaje se da en el programa de Ingeniería Electromecánica de la Universidad Autónoma de Colombia. Es esta una actividad de aprendizaje basada en proyectos, en la que la metodología de los proyectos integrados de semestre constituye un proceso dinámico que año tras año ha exigido el esfuerzo del equipo docente en su función de orientación y acompañamiento, y ha requerido ajustes como resultado de la evaluación permanente del proceso; en la actividad de aprendizaje, el examen documental de los proyectos realizados devela un testimonio importante acerca de conocimientos construidos en las áreas de énfasis del programa, de las habilidades y destrezas logradas por el estudiante en técnicas de diseño y de investigación, y fue significativa la cantidad de proyectos destacados por su contenido social, por su ingrediente de investigación, por su grado de interdisciplinariedad y/o por la aplicación rigurosa de elementos metodológicos de diseño. No obstante, lo más destacable es que detrás de cada proyecto siempre hubo un proceso intenso de trabajo del estudiante, de indagación, de interacción, de compenetración con problemas reales de la comunidad. Por encima incluso de la misma calidad del proyecto realizado, el hilo conductor siempre ha sido el corroborado semestre tras semestre, valor agregado como método de autoformación guiada del estudiante para su desarrollo integral [9].

Otra experiencia que vale la pena mencionar es la de la Universidad de Deusto, España, la cual permitió mostrar cómo es posible diseñar y aplicar un aprendizaje basado en competencias y organizado aplicando el proyecto integrador, así como demostrar que los resultados de estos proyectos son útiles y valiosos para producir mejoras apreciables y significativas, tanto en la docencia del profesor, como en el aprendizaje de los estudiantes, contribuyendo a la mayor satisfacción y bienestar de ambos al implicarse en las actividades de docencia y aprendizaje [10].

Otro ejemplo en España es la Universidad de Málaga, con el proyecto integrador "Creando Empresas”. Este lo desarrolla el Departamento de Economía con el objetivo de que las alumnas y alumnos adquieran las siguientes capacidades: 1) obtener una visión práctica del funcionamiento de una organización empresarial a través de las diferentes áreas que la componen; 2) adquirir los conocimientos necesarios para emprender un proyecto empresarial de pequeña escala; 3 ) identificar y evaluar los factores externos que condicionan el proyecto de iniciativa empresarial; 4) conocer los distintos documentos que genera el desarrollo de la actividad empresarial; 5) analizar las diferentes formas jurídicas vigentes de empresa, señalando la más adecuada en función de la actividad económica y los recursos disponibles; 6) adquirir la capacidad para llevar a cabo la puesta en marcha de una empresa; 7) conocer las normas sobre la obligación de conservar la documentación que la empresa genera; 8) realizar previsiones de tesorería para conocer su situación a corto plazo, y prever los posibles problemas de falta de liquidez; 9) fomentar la cultura emprendedora y el trabajo en equipo; y 10) promover el uso de las Tic [11].

Otro ejemplo es la Universidad de Sonora en México, la cual propone proyectos integrados de aprendizaje para matemáticas, ejemplo que muestra cómo los contenidos matemáticos pueden estudiarse y aprenderse utilizando didácticas que inciden directamente en la comprensión, por parte del alumno, de la necesidad de estudiar y aprender matemáticas, puesto que le ven la utilidad de manera inmediata al resolver problemas reales con los que se pueden enfrentar en el futuro. Además, se favorece el gusto por el aprendizaje de las matemáticas, y a su vez se desarrollan habilidades y destrezas diversas, tanto técnicas, como sociales [12].

Una de las experiencias de interés en este ejercicio de estado del arte presentado por Filho y otros en el Journal of Cleaner Production, en el artículo "Implementing and operationalising integrative approaches to sustainability in higer education: the role of Project-oriented learning" [13], enfatiza la importancia que tiene la función del aprendizaje orientado a proyectos como una herramienta que apoya los enfoques integradores de dicho aprendizaje, dentro del contexto del desarrollo sostenible y los problemas de sostenibilidad; es decir, dicho aprendizaje por proyectos debe tener una amplia utilidad al momento de apoyar los enfoques integradores para la sostenibilidad, y por ello tiene que ser una práctica de las instituciones de educación superior, y se presenta como una tendencia mundial.

En esta línea, se ha recolectado el trabajo realizado por Nicoleta Duta de la Riveras de la Universisdad 
de Rumnania, y de Oscar Martínez-Rivera, de la Universidad de Barcelona, en el artículo "Between theory and practice: the importance of ICT in Higer Education as a tool for collaborative learning" [14]. De este cabe mencionarse que en programas de e-learning, así como en programas de sostenibilidad, se constituyen tres ciclos. Se inicia por el curso de graduación, el cual involucra 20 unidades curriculares obligatorias entre disciplinares y básicas; se pasa al segundo ciclo de maestría con la temática de particiapción ambiental ciudadana como un programa de b-learning que desarrolla competencias en el área de desarrollo sostenible, con aspectos ambientales y sociales; luego, se llega al grado de doctorado en sostenibilidad social y desarrollo. Los importantes niveles de satisfacción evindenciados en los estudiantes incluyen la relevancia y el valor que tiene el alimentar su propia investigación dentro de los tres ciclos, a fin de demostrar la integridad de los conocimientos adquiridos en todo el trabajo transversal que se realiza dentro de la formación que contempla los tres ciclos mencionados.

En su carácter resolutivo, los proyectos integradores buscan resolver problemas del contexto, bien sean del sector gubernamental, industrial, comercial y/o de servicios, mediante la implementación de soluciones que incorporen las competencias de las diferentes asignaturas del programa de estudio. Al participar en proyectos integradores durante su formación profesional, el estudiante aprende a aprender, aplica conocimientos para la resolución de problemas, desarrolla actividades de investigación y tiene una visión interdisciplinaria. Estos proyectos constituyen experiencias vivenciales que permiten desarrollar las competencias específicas y genéricas (estas últimas conforman los aspectos deseables para las empresas, tales como el compromiso ético, el liderazgo, el trabajo en equipo y la capacidad de comunicación oral y escrita, entre otras muy importantes).

Como estrategia curricular, los proyectos integradores se pueden implementar por gran diversidad de proyectos, pero se pueden generalizar en función de cuatro aspectos centrales de un diseño curricular con enfoque en competencias, lo cuales se dan de acuerdo con (a) las competencias que se enfatizan, (b) la relación que se establece con las disciplinas, (c) su enfoque, y (d) el grado de participación de los estudiantes.

Cuando el proyecto a realizar se plantea en un conjunto de acciones que direccione el desarrollo de las competencias genéricas establecidas en un plan de estudios, entonces se trata de un proyecto genérico; en cambio, cuando el direccionamiento se enfatiza en las competencias específicas de dicho plan, entonces es un proyecto específico; por último, cuando se direccionan de manera integral las competencias genéricas y específicas del plan de estudios, entonces se hace referencia a un proyecto global. Ahora bien, si el proyecto se integra por un conjunto de asignaturas de un mismo plan de estudios, se trata de un proyecto disciplinario; en cambio, cuando el proyecto integre un conjunto de asignaturas de diversos planes de estudio, será un proyecto interdisciplinario [6].

En cuanto al desarrollo del proyecto, cuando se orienta al estudiante: (a) a que aprenda a organizar, sistematizar y analizar la información de un hecho para obtener conclusiones que puedan enriquecer el campo de conocimiento, se habla de un proyecto de investigación; (b) al diseño, construcción y transferencia de tecnología (equipos, instrumentos, procesos, entre otros), entonces es un proyecto tecnológico; (c) a identificar, diagnosticar y resolver problemas sociales vinculados con la comunidad y su entorno, es un proyecto social; (d) a emprender proyectos para generar autoempleos y economías, es un proyecto económico; y (e) a crear o recrear mediante estrategias recreativas, deportivas y artísticas, es un proyecto cultural [6].

Finalmente, cuando el estudiante desarrolla sus competencias a través de un proyecto, su nivel de alcance en el desempeño de las competencias puede ser diferente, es decir, su nivel de participación puede variar. Si en esta participación el estudiante solo se limita a ejecutar el proyecto sin la realización de sugerencias, mejoras o trabajo colaborativo, entonces se habla de un nivel operativo; en cambio, cuando proponen la estructuración y construcción de las acciones a realizar durante la ejecución del proyecto, se dice que es un nivel intermedio; y si el estudiante es capaz de proponer un proyecto en conjunto con el docente o de modificar uno ya existente, entonces se encuentra en un nivel estratégico [6].

De acuerdo con López-Rodríguez [6], las fases recomendadas para el desarrollo de un proyecto son: contextualización/diagnóstico, fundamentación (marco referencial), planeación, ejecución, evaluación y socialización.

Lo primero que se debe llevar a cabo es una aproximación y reconocimiento de la realidad del objeto de estudio, es decir, un diagnóstico 
(contextualización/diagnóstico). Con base en este diagnóstico se constituye un marco referencial teórico que fundamenta el proyecto (fundamentación). Luego, se realiza el diseño del proyecto mediante la aplicación de cualquier método de proyectos (planeación), y se desarrollan cada una de las actividades planeadas (ejecución), para después realizar un reconocimiento de logros y aspectos a mejorar en el proyecto (evaluación). Por último, se lleva a cabo un diálogo, una reflexión y un análisis de los diversos conceptos, aplicaciones y experiencias alcanzadas durante el proyecto integrador (socialización).

La revisión de los proyectos integradores demanda una mirada colectiva en la que los docentes de las asignaturas específicas revisan los aspectos centrales a nivel teórico, conceptual, procedimental y metodológico, en relación con el tema específico abordado. Los docentes de las asignaturas transversales evalúan los aspectos generales que debe involucrar el proyecto, independientemente de la especialidad, relacionados con el manejo y la búsqueda de información, su validez y confiabilidad, los impactos éticos que tienen los planteamientos para las comunidades y el ambiente, así como su componente emprendedor [15].

Instituciones como la Universidad Interamericana de Puerto Rico, la Tecnológica América unita de México, la Universidad ort de Uruguay, la Tecnológica Nacional de Buenos Aires, la Nacional de Córdoba de Argentina [16], y en Colombia, la Universidad del Cauca, la San Buenaventura y la Fundación Academia de Dibujo Profesional de Santiago de Cali, entre otras, han incorporado iniciativas relacionadas con el desarrollo de proyectos integradores, en el marco de procesos investigativos o como actividades de aula.

Las definiciones revisadas en este apartado se retomarán con el fin de adaptarlas y redefinirlas con respecto a los planes de estudio diseñados en el enfoque de competencias profesionales en lo tecnológico, porque se pretende proponer, desde el aula de clase, soluciones innovadoras para la formulación y desarrollo de proyectos integradores, direccionados a las problemáticas del entorno.

El esfuerzo más importante de la experiencia pedagógica está centrada en lograr que los estudiantes hagan una apropiación profunda del conocimiento y su marco conceptual, a fin de direccionar el verdadero sentido que tiene la formulación y el desarrollo de los proyectos integradores, así como romper con los idearios estudiantiles en los que existe una tendencia a creer que el esfuerzo académico en talleres y en el trabajo independiente se limita a un cumplimiento de clase exigido por el docente para asignar una nota, lo cual no tiene trascendencia fuera de ella.

Este argumento evidencia la importancia que tiene la conceptualización de cinco factores claves de la construcción de la experiencia pedagógica, entendidos sobre las siguientes bases teóricas:

El educador. Como orientador de la práctica pedagógica denominada "proyecto integrador", motiva al estudiante en la competencia propositiva y lo acompañan en su propuesta de proyecto logrando integrar los saberes interdisciplinarios. Sin embargo, el educador en esta experiencia pedagógica debe combinar un conjunto de perfiles propios de su quehacer, asociados en primer lugar a ser formador a través de la transferencia de conocimiento y experiencia propios del emprendimiento y la innovación. En segundo lugar, a ser facilitador, con lo que contribuye a despertar en el estudiante una vocación emprendedora y, a su vez, a proporcionar los instrumentos que hagan viable su labor estudiantil; en tercer lugar, ha de ser un promotor que pone a prueba la iniciativa empresarial de los estudiantes [17].

Aprendizaje. Significa que todo el engranaje que conlleva el proyecto integrador se encuentra basado en un marco teórico, en el que se "deben considerar dos cosas básicas [...] en primer lugar realizar investigación y en segundo lugar asegurarse de que los estudiantes entienden las bases teóricas y conceptuales que expliquen por qué algunos emprendedores tienen éxito y otros fracasan" [18]. Es decir, que el aprendizaje no solo consiste en apropiarse de conjuntos de saberes, sino que se deben buscar en el entorno, argumentos experimentales sustentables que los estudiantes deben adaptar en sus trabajos. Eso quiere decir que el aprendizaje debe ser una mezcla de la teoría que intelectualmente haga progresar al estudiante, y de análisis de casos y aplicación práctica, lo que les permita estudiar el éxito y el fracaso, así como actuar con base en decisiones. Esta es la base de la teoría de enlace entre teoría y práctica, desarrollada por Bill Bygrave, director del centro de emprendimiento de Babson College en Massachusetts, Estados Unidos [18].

Proyecto integrador. Estrategia pedagógica de aula que permite a los estudiantes aplicar los saberes interdisciplinarios a fenómenos del entorno, mediante la orientación del docente, de manera 
que capacite a los estudiantes para elaborar propuestas de solución que puedan contener elementos innovadores y un componente básico de formación investigativa. En este sentido, se menciona que el objetivo del proyecto integrador [19] es fomentar en estudiantes y docentes un espíritu investigativo que parta de la experiencia proporcionada por la realización de un trabajo en equipo, evidenciando la integración de los saberes relacionados con cada disciplina de estudio de un semestre específico.

Innovación. Se constituye en factor fuerte no solo dentro de los retos que deben asumir las empresas actualmente para mejorar sus niveles de productividad y competitividad, sino, más desafiante aún, para las universidades, las cuales deben asumir un rol muy proactivo de relación con el entorno empresa, gobierno y sociedad; de ahí la importancia que toma el desarrollo de la investigación, ya sea básica o aplicada, pues ambas son útiles a ese entorno, y la extensión, dentro de la proyección social y el aporte al empresariado, en el contexto en que dichas funciones están totalmente ligadas al componente transversal de la innovación. Si se hace una indagación minuciosa del concepto de innovación, actualmente los expertos consideran que es necesario apuntar a un enfoque denominado "Sistema nacional de innovación", que hace su aparición como resultado de la fusión de varios enfoques y conceptos: primero se basa en el concepto de los distritos industriales desarrollado por Marshall, en la primera mitad del siglo $\mathrm{xx}$, también lo hace en la teoría de los polos de desarrollo generada principalmente por Perroux en la década de los cincuenta y por último, en la teoría de los clusters de Porter, desarrollada en la década de los noventa a partir de la publicación de las ventajas competitivas de las naciones. Estos enfoques tienen varios elementos en común, como la importancia que adjudican a la proximidad espacial, las externalidades, la cultura e identidad, y el proceso de aprendizaje colectivo. Según Romer y Lucas, las innovaciones generan externalidades positivas, las cuales pueden ser aprovechadas por los agentes de una nación, región o localidad [20].

En otras palabras, la innovación es el componente altamente generador de los verdaderos impactos que se producen en la sociedad, siempre y cuando se encuentren articulados el conocimiento y la experiencia de los actores al conjunto de efectos de bienestar esperados por la comunidad como un todo.
Emprendimiento. En el contexto mencionado que define de alguna manera el concepto de innovación, el emprendimiento también está unido al gran reto de globalización que está enfrentando a las empresas y los emprendedores, es decir, en lo que se ha querido denominar, no una era de cambios, sino un cambio de era. Dadas las fuertes transformaciones que se han suscitado en el ámbito geotecnológico, es claro que ambos actores, más allá de preocuparse por fabricar de modo ordenado productos de calidad, deben focalizarse en fabricar ideas nuevas y generar innovación de modo sistemático, porque es la manera en que se están jugando el futuro las compañías, y quienes asumen con actitud de éxito el riesgo financiero, para mitigar el grado de incertidumbre y obtener las mayores ganancias personales y empresariales.

Ahora bien, la universidad está impulsando su actividad formadora en el emprendimiento, de manera que los programas académicos están modificando la transversalidad del impacto de la formación, promueven la cultura del emprendimiento en el trabajo de aula o proyecto integrador, el cual pretende alimentar las competencias investigativas y a la vez el conocimiento del entorno en el estudiante, con el fin de facilitar la aplicación del conocimiento y la creatividad en la construcción de ideas emprendedoras e innovadoras que transcurran en un proceso de maduración desde los primeros semestres hasta su proyecto de grado. Esto, luego, a fin de que como egresado continúe en esa contribución de soluciones alternativas que proporciona el aprendizaje y la aplicación de saberes.

De acuerdo con lo anterior, el programa OECD LEED y la Comisión Europea han sugerido en Una guía de trabajo para Universidades Emprendedoras, [21, p. 1] siete factores claves mediante los cuales la universidad debe evaluar la permanencia del nivel de emprendimiento: "1) liderazgo y gobernanza; 2) capacidad organizacional, personas e incentivos; 3 ) desarrollo del emprendimiento en la docencia y la investigación; 4) trayectorias para los emprendedores; 5) relación universidad-empresa para el intercambio de conocimiento; 6) internacionalización; 7) medición del impacto" [22]

Lo anterior orienta a la necesidad de visibilizar un líder capaz de alcanzar la eficacia, la calidad y la buena orientación del ejercicio de docencia, investigación y extensión, direccionado al emprendimiento y la innovación. 


\section{Metodología}

El proyecto integrador (PI) surge como una estrategia pedagógica para que el estudiante aplique los conocimientos aprendidos en clase, y reconozca la importancia y utilidad de sus aprendizajes en el campo laboral y el entorno empresarial.

De esta manera, un proyecto integrador debe representar el vértice de los procesos claves de la UDES como lo son: docencia, investigación y extensión (según el proyecto educativo institucional-PEI), y debe orientar y promover el fortalecimiento de las capacidades de investigación y de innovación de la institución.

Es necesario que todo el cuerpo docente esté involucrado en la realización del proyecto integrador que se planea para cada semestre; de su nivel de compromiso dependerá la obtención de resultados exitosos.

\subsection{Direccionamiento}

Para el desarrollo del proyecto se han identificado cuatro etapas:

Inicio del proyecto. El docente, en el rol de guía del proceso de aprendizaje, explica la dinámica del proyecto integrador, así como la importancia que tiene para el aprendizaje y puesta en marcha del componente práctico. Los estudiantes tienen participación activa una vez exponen sugerencias para el desarrollo del proyecto, las cuales serán tomadas por el docente y así, en un diálogo constructivo, se establecen estrategias que finalmente serán consignadas junto con las directrices del docente en un acta de inicio de clase, en la que se incluye lo acordado en el proyecto integrador: estrategias, identificación de productos a mejorar, definición de la necesidad en cada producto, tipificación de los insumos que contribuyen con la mejora, caracterización del proceso de mejora y determinación del valor agregado en el producto.

Desarrollo del proyecto. De acuerdo con la planeación, los estudiantes empiezan a desarrollar el proyecto integrador teniendo en cuenta que deben articular los conocimientos desde las diversas asignaturas. En el caso de los estudiantes de cuarto semestre del programa de Tecnología Industrial, la asignatura integradora es métodos y tiempos para trabajar un núcleo problémico, a partir de la propuesta que los estudiantes deben plantear simulando la producción de un producto que debe incorporar un componente innovador; por ejemplo [23], un trabajo de grado que se desarrolló a partir de un proyecto integrador en el cual los estudiantes identificaron que las tapas de botellas plásticas residuales producen impactos ambientales importantes y plantean un uso alternativo, al proponer la producción de enchapes con dichas tapas. A partir de este evento de simulación, se articulan las otras asignaturas en las que deben aplicar los conocimientos en salud ocupacional, contabilidad gerencial y logística, logrando así obtener finalmente el tiempo estándar para producir una pieza de enchape, las medidas de seguridad en el trabajo, el costo del producto y el empaque, el embalaje y el almacenamiento del mismo (véase la tabla 2).

Es importante destacar que el ejercicio práctico mencionado aborda dos aspectos metodológicos claves. El primero apropia el aprendizaje basado en problemas [24] porque el estudiante apropia la situación problema en un contexto de equipo y trabajo colaborativo que involucra un método dinámico, creativo y eficaz, el cual utilizará el docente como medio de revisión en el siguiente paso. El segundo aspecto metodológico adecúa el aprendizaje orientado a proyectos [23], porque aborda

Tabla 2. Asignatura integradora y asignaturas articuladoras en cuarto semestre

\begin{tabular}{|l|l|l|l|}
\hline Código & \multicolumn{1}{|c|}{ Nombre } & \multicolumn{1}{|c|}{$\begin{array}{c}\text { Clase de asignatura } \\
\text { (integradora / articuladora) }\end{array}$} & $\begin{array}{c}\text { Función del docente de la } \\
\text { asignatura } \\
\text { (gerente / articulador) }\end{array}$ \\
\hline 49204 & Contabilidad Gerencial & Articuladora & Articulador \\
\hline 49214 & Métodos y tiempos & Integradora & Gerente \\
\hline 49224 & Salud Ocupacional y Seguridad Industrial & Articuladora & Articulador \\
\hline 49911 & Logística & Articuladora & Articulador \\
\hline
\end{tabular}

Fuente: elaboración propia 
el aprendizaje experiencial en torno a un tópico, orientado a la resolución de problemas complejos a partir de soluciones abiertas, con las siguientes características:

- Está centrado en el alumno.

- Parte de un planteamiento que se basa en un problema real y que abarca distintas áreas.

- Apoya contenidos académicos y presenta propósitos auténticos.

- Ofrece oportunidades para que los estudiantes realicen investigaciones que les permitan aprender nuevos conocimientos, aplicar la información y representar su conocimiento en diversas formas.

- Tiene metas educativas explícitas.

- Se basa en el constructivismo (teoría de aprendizaje social).

- Promueve la colaboración y el aprendizaje cooperativo, involucrando a los alumnos mediante el compromiso en la elaboración de un producto.

- El profesor actúa como facilitador.

Estas características alimentan la perspectiva del estudiante con el propósito de consolidar el proyecto integrador en el trabajo de grado para optar al título profesional, como ocurrió con el trabajo de las tapas plásticas, así como con un ejercicio posterior que partió del uso alternativo de las botellas PET residuales para la producción de ladrillo en el sector de la construcción en condiciones de sostenibilidad [25].

El componente innovador en el desarrollo del proyecto integrador se incorpora sobre la base de la acción creativa y de cambio que realiza el estudiante, al incluir algo nuevo dentro de la realidad existente, en cuya virtud esta resulta modificada, así como el resultado de la actividad misma [26]. Es decir, innova tanto el proceso de transformación de la materia prima, como el producto final, con el valor agregado resultante de dicha transformación.

Una vez concluido el semestre en el que se desarrolla el proyecto integrador, los estudiantes ingresan al semillero de investigación para seguir con el desarrollo de la idea, y de esta manera dar cumplimiento a la rigurosidad que requieren los procesos investigativos.

Revisión de resultados preliminares. El docente realiza una revisión del avance y la calidad de los proyectos, basado en lo que el estudiante ha aprendido en su praxis, más allá de los saberes conceptuales; es decir, se trata de aplicar un sistema de evaluación preliminar que permita valorar en el estudiante lo alcanzado en los conocimientos y las competencias que se definieron previamente [27] en las asignaturas articuladoras. Dado que el programa Tecnología Industrial realiza la semana de Innoempres, en la que una de sus actividades contempla la presentación, en un escenario institucional, de los mejores proyectos integradores, el docente clasifica los proyectos meritorios y les informa que han sido seleccionados para la posterior presentación.

Presentación de resultados y lecciones aprendidas. Por último, los estudiantes socializan ante el comité curricular del programa los resultados finales y se establece un conversatorio sobre lecciones aprendidas. Durante la semana de Innoempres se presentan solo los proyectos meritorios. Al observar los resultados, se puede corroborar que el saber tecnológico está orientado a la acción, es decir, a la resolución de problemas en diversos campos de aplicación, conformándose una fuente de conocimiento de las acciones en la realidad; de esta manera, los saberes del tecnólogo posicionan su conocimiento para conocer la realidad y proponer alternativas de solución a problemas identificados en el logro de objetivos esperados.

Asimismo, es importante tener en cuenta que dentro de los roles del proyecto integrador se encuentra la asignatura integradora, la asignatura articuladora, el docente gerente del proyecto y el docente articulador.

Asignatura integradora. Es aquella asignatura que tiene como función principal articular las actividades que se llevan a cabo en las demás asignaturas del semestre [17].

Asignatura articuladora. Son asignaturas de apoyo; sirven de soporte durante el semestre al brindar herramientas metodológicas e investigativas que aportan a la consecución de los objetivos fijados por la asignatura integradora.

Docente gerente de proyecto. Es el docente responsable del proyecto integrador; se caracteriza por su conocimiento disciplinar e investigativo, debe promover la creatividad e innovación y es quien tiene a cargo la asignatura integradora.

Docente articulador. Son los docentes que tienen a cargo las asignaturas articuladoras. Su función es concretar, apoyar y consolidar el proyecto integrador del semestre desde sus materias, y de 
acuerdo con el documento entregado por el docente gerente de proyecto.

Contenido del proyecto integrador. Los trabajos se presentan de acuerdo con los lineamientos establecidos por la norma Icontec 1486.

\section{Resultados}

La experiencia presentada demuestra la importancia de una estructuración robusta de planeación, dirección, ejecución y control en la gestión que se realiza con el proyecto integrador, como estrategia pedagógica en el aula de clase que fortalece las competencias del estudiante de tecnología.

\subsection{Planeación del proyecto}

A fin de ejecutar las directrices presentadas en el punto anterior, el comité curricular se reúne al final de cada semestre para mostrar las experiencias exitosas, pero también las lecciones aprendidas. Esto con el propósito de realizar un permanente mejoramiento en el proceso; de esta manera, se realiza una jornada de trabajo a fin de establecer las nuevas directrices para el desarrollo del proyecto integrador del semestre en curso y las estrategias de implementación.

En este sentido, se ha trabajado con el formato indicado en la tabla 3 con el objetivo de establecer las estrategias.

Tabla 3. Formato de estrategias

\begin{tabular}{|l|l|l|l|}
\hline $\begin{array}{c}\text { Actores que } \\
\text { intervienen/ } \\
\text { aspectos por } \\
\text { mejorar } \\
\text { Estudiantes }\end{array}$ & Solución & $\begin{array}{c}\text { Estrategia } \\
\text { de imple- } \\
\text { mentación }\end{array}$ & $\begin{array}{c}\text { Indicador de } \\
\text { seguimiento }\end{array}$ \\
\hline Docentes & & & \\
\hline Universidad & & & \\
\hline
\end{tabular}

Fuente: Comité Curricular Programa de Tecnología Industrial

A continuación, se expone un ejemplo de cada uno de los aspectos.

\subsubsection{Estudiantes}

Aspecto por mejorar. Estudiantes dicen no tener tiempo para dedicar al desarrollo del proyecto, dado que es una población que estudia en la noche, trabaja en el día y la mayoría son padres de familia.

Solución. Disminuir el tiempo de desarrollo del proyecto en horas de trabajo independiente, e incluirlo en horas directas.

Estrategia de implementación. Durante la clase orientará la teoría, y los ejercicios prácticos se desarrollarán directamente sobre el proyecto integrador.

Indicador de seguimiento. Con el fin de medir la eficiencia de este indicador, se tendrá en cuenta la calidad con que se desarrolla el proyecto, dado que se aprovechará la asesoría del docente durante la clase, de modo que queda así: "número de avances de proyectos evaluados/ número de ellos que son evaluados con nota superior a 3,5 ".

\subsubsection{Docentes}

Aspecto por mejorar. No tienen claridad en la finalidad del proyecto integrador.

Solución. Capacitar al docente en el tema.

Estrategia de implementación. Una semana antes del inicio de clases se orienta una charla sobre concepto, objetivos y finalidad del proyecto integrador, así como directrices a seguir, se reconfirma el compromiso con el desarrollo del proyecto y se define el núcleo problemático a resolver.

Indicador de seguimiento. $\mathrm{N} .^{\circ}$ de docentes capacitados/N. ${ }^{\circ}$ de docentes del programa.

\subsubsection{Universidad}

Aspecto por mejorar. Hace falta detallar la planeación del desarrollo de proyectos integradores.

Solución. Realizar planeación detallada del proyecto integrador.

Estrategia de implementación. En consenso con los profesores y estudiantes se establecerá la metodología y la planeación.

Indicador de Seguimiento. Número de participantes en el taller/número de personas convocadas.

Documento que evidencie la planeación semestral del proyecto integrador.

\subsubsection{Cronograma y recursos}

Se ha estipulado que el proyecto integrador valga la totalidad de la tercera nota de la asignatura, pero deben realizarse tres entregas durante el semestre, las cuales serán acumulativas para dicha nota. De esta 
manera, tiene un componente motivacional para el estudiante y para el docente, dado que se trabaja activamente en busca de un buen resultado final que será presentado al terminar el semestre. Las entregas están programadas de la siguiente manera:

- Primera entrega: 26 de septiembre, correspondiente al $20 \%$ de la nota de $\mathrm{P} 3$.

- Segunda entrega: 24 de octubre, correspondiente al $30 \%$ de la nota de $\mathrm{P} 3$.

- Tercera entrega: 14 de noviembre, correspondiente al $50 \%$ de la nota de $\mathrm{P} 3$.

Para el desarrollo del proyecto es indispensable la continua orientación del docente. En este caso, se requiere el uso de salones, materia prima para simular la producción, visita a empresa y uso de audiovisuales, así como uso de software que ayuda a diagramar los procesos de hombre, máquina y alistamiento para la presentación final.

\subsubsection{Ejecución del proyecto}

Con las premisas dadas anteriormente, y teniendo en cuenta las fechas de los entregables, se procede a desarrollar el trabajo planeado que está distribuido entre las horas de trabajo directo y las horas de trabajo independiente.

A fin de facilitar el desarrollo del aspecto práctico de las asignaturas, se aplican los talleres directamente sobre el proyecto integrador, permitiendo así un avance mucho más rápido y disminuyendo la dedicación al proyecto con respecto a las horas de trabajo independiente, que es uno de los aspectos que dificultan el éxito de esta experiencia. Sin embargo, el docente planea el trabajo independiente para que los estudiantes lleven otros avances en las próximas clases, y así propiciar que el desarrollo del proyecto sea realmente eficiente.

\subsubsection{Socialización y evaluación final del proyecto}

La evaluación en el proyecto es un proceso permanente, y es en la entrega final cuando se asigna una mayor ponderación. La socialización se realiza en dos momentos: la primera se realiza frente al comité curricular, y es cuando se reconfirman cuáles son los proyectos meritorios; la segunda socialización se realiza durante la semana de Tecnología
Industrial (Innoempres), y tiene como finalidad evidenciar el impacto que ha tenido el desarrollo del proyecto, las lecciones aprendidas, las oportunidades de mejora y de emprendimiento. Esto, en razón a que los productos finales hacen referencia a productos innovadores que pueden ser presentados en el Fondo Emprender, o ante entes que promuevan el emprendimiento.

\subsubsection{Productos finales}

Los trabajos presentados se han destacado por la apropiación del proyecto integrador por parte del estudiante, estimulando su creatividad en la elaboración del prototipo de su propuesta. El conjunto de prototipos se dona a una comunidad rural de Bucaramanga, como un evento de proyección social [28], lo que fortalece la alianza de la formación, la investigación y la extensión que incluye la gestación de su propio trabajo de grado. A continuación, se realiza una breve presentación de ideas que se recrearon como un prototipo y aquellas que se constituyeron en trabajos de grado.

Salas ecológicas con base en llantas recicladas. Las llantas automotrices residuales representan un grave y creciente problema ambiental derivado de las actividades humanas; actualmente, está restringida su disposición en los rellenos sanitarios y su frecuente almacenaje a la intemperie implica un alto riesgo, tanto a la salud, como al medio ambiente y a la seguridad de la población. Su estructura compleja, formada por diversos materiales tales como caucho, acero y tejido de poliamida o poliéster, permite ser utilizada como materia prima para diversos procesos de reutilización (como, por ejemplo, la fabricación de muebles), puesto que a partir de llantas enteras que poseen la ventaja de procesarlas con requerimientos de mínima cantidad de aplicación de energía y un costo muy bajo, se facilita su manufactura en instalaciones básicas. La competencia de mayor fortalecimiento en los estudiantes fue en lo procedimental (hacer) por medio del estudio y aplicación de métodos, técnicas, procedimientos, herramientas e instrumentos reconocidos por la comunidad académica y profesional. En el proyecto integrador participaron cuatro estudiantes que iniciaron su trabajo a partir del semestre 1 del 2014, y el producto hizo parte del evento de proyección social realizado con una comunidad que pertenece a un nivel de estratificación 1, aledaña a la Universidad de Santander. 
Forro para cojín de moto. El producto consiste en un forro protector para el cojín de motocicleta. Se caracteriza por ser impermeable y térmico, y tiene como objetivo minimizar el impacto causado por la exposición a los rayos solares y a la lluvia. Está fabricado con materias primas de alta calidad como aislante térmico, material impermeable y tela dril. Este forro protector contará con diversos diseños y estilos, debido a la gran diversidad de estructuras y formas en los cojines de las motos. Con el propósito de satisfacer el gusto de hombre y mujeres, estará fabricado en diferentes colores para todos los gustos. Principalmente se reforzaron las competencias, tanto intelectuales, como prácticas, en el trabajo de equipo. De la misma manera, la competencia del hacer en la aplicación de técnicas y procedimientos. En este trabajo de proyecto integrador participaron cinco estudiantes quienes iniciaron su trabajo a partir del semestre 1 del 2014, cuando cursaban cuarto semestre de carrera y el producto se entregó en el evento de proyección social a finales del 2015, realizado con una comunidad que pertenece a un nivel de estratificación 1, aledaña a la Universidad de Santander.

Plan de negocio para la creación de una empresa productora y comercializadora de muebles en madera aglomerada y tubos de cartón en Bucaramanga. El alto volumen de residuos de madera y cartón producido por la industria en general, y en las carpinterías en particular, contribuyen significativamente al impacto ambiental. Además, es conocido que la madera como materia prima es vulnerable a la humedad y los insectos, lo que debilita su uso alternativo como residuo. En este sentido, el aglomerado de madera es una alternativa de uso de los residuos de esta materia prima que puede ser utilizada para la fabricación de muebles, combinada con tubos de cartón residual, lo que permitió como resultado del trabajo, un prototipo de silla para niños de tres años de edad que resulta de fácil construcción y económicamente bajo en costos. La propuesta de plan de negocios se orienta a pequeños empresarios de la ciudad que tienen fortalezas como fabricantes de muebles. La competencia que se fortaleció fue la argumentativa, al explicar razones y motivos sobre la solución al problema planteado, teniendo como base el contexto disciplinar y metodológico. El trabajo realizado por cuatro estudiantes que cursaban cuarto semestre de carrera en el segundo semestre del 2014, se orientó a la asesoría que presta el SENA a través de la unidad de Tecno-parques, a fin de ser presentado en el Fondo Emprender de dicha entidad estatal.

Diseño de plan estratégico y análisis del mercado para la cervecería artesanal Bier Krug sas. La pequeña empresa tiene la tendencia a mantener su quehacer de manera informal, por lo que carece de herramientas que les permita estructurar una gestión empresarial robusta. Esto llevó a que un grupo de cinco estudiantes del semillero Ágora de Tecnología Industrial, propusiera el diseño del marco estratégico y de mercadeo mediante la identificación de variables externas e internas que afectan la operatividad del negocio de la cerveza artesanal, implementando las estrategias necesarias para el buen desarrollo de la cervecería Bier Krug. Además, se verificó el cumplimiento de los estándares mínimos requeridos para la obtención de la licencia de funcionamiento del Invima. Este ejercicio se transformó en trabajo de grado, y sirvió de base a uno de los estudiantes para que, junto con su familia, constituyeran la empresa Bier von Eder, considerada una de las empresas de cervecería artesanal más importantes de la región. El mayor reconocimiento se dio a través del consumidor turístico en la mesa de Los Santos, Santander, y se trabajaron las competencias, tanto argumentativas, como procedimentales, que reflejaron el esfuerzo de los estudiantes en este trabajo en explicar razones y motivos sobre la solución del problema planteado y el estudio y aplicación de métodos, técnicas, procedimientos, herramientas e instrumentos reconocidos por la comunidad académica y profesional, a fin de completar la solución proyectada.

Ekoentapes un enchape a base de materiales reciclados para el revestimiento de muros en el área de acabados del sector de la construcción. Creación y producción de un enchape innovador que contribuya con el medio ambiente para ser utilizado en el área de acabados en la construcción de vivienda, elaborado a partir de tapas plásticas como materia prima, ya que es este uno de los desechos más abundantes en el mundo y uno de los principales generadores de contaminación, debido a su difícil degradación. Por esta razón, la reutilización de estos materiales contribuirá a la protección del medio ambiente y al desarrollo de la responsabilidad social empresarial. El trabajo permitió reforzar especialmente la competencia propositiva al proponer hipótesis para explicar determinados hechos, construir soluciones a problemas, así como escoger la alternativa de solución más eficaz y eficiente 
para el problema planteado. Las cuatro estudiantes que realizaron el trabajo también iniciaron el desarrollo de la idea en cuarto semestre de la carrera en el primer semestre del 2014, y tuvieron la oportunidad de promover la idea a través del semillero de investigación Ágora del programa de Tecnología Industrial. La idea fue presentada a la convocatoria interna de semilleros de investigación, y dada su acogida, tuvo como resultado de dicha convocatoria haber sido seleccionada para la regional de semilleros de investigación, en la cual también obtuvo un buen nivel de aceptación.

Plan de negocios para la producción y comercialización de ladrillos ecológicos- ecoladrillo. Este proyecto tiene por objeto incorporar residuos plásticos en la industria de la construcción para la sustitución de agregados como el hormigón común, a fin de obtener un producto sostenible que supere las características de los materiales de aislamiento térmico tradicionales. En este trabajo se aplicó una metodología de investigación-acción integral, en el que los resultados de los materiales investigados se caracterizan con base en factores tales como la densidad, la absorción de agua, la conductividad térmica, la resistencia mecánica, el envejecimiento, la resistencia al fuego y la permeabilidad al vapor de agua. Al igual que en el trabajo anterior, la competencia propositiva fue pertinente al proponer hipótesis que permiten explicar determinados hechos, construir soluciones a problemas y escoger la alternativa de solución más eficaz y eficiente para el problema planteado. Esto se aunó a la competencia procedimental que permitió utilizar los métodos, las técnicas y las herramientas reconocidas por la comunidad académica. El trabajo realizado por cinco estudiantes que cursaban cuarto semestre de carrera en el segundo semestre del 2014, se orientó a la asesoría que presta el sENA a través de la unidad de Tecno-parques, y ha avanzado en la evaluación financiera para ser presentado en el Fondo Emprender de dicha entidad estatal.

Este conjunto de productos han sido desarrollados en el lapso de cuatro periodos académicos, con la participación de alrededor de 27 estudiantes, comenzando desde el 2014. Aunque no iniciaron al tiempo, fueron desarrollados de manera secuencial, ya que tuvieron la oportunidad de presentarse en escenarios de convocatorias de semilleros de investigación. Esto dentro del ejercicio de proyección social con los productos que se lograron materializar y se entregaron en donación a una comunidad del entorno de la universidad. Cabe recordar que los estudiantes cursaban su cuarto semestre de carrera, es decir, casi en la mitad de su formación maduran un proceso que en la mayoría de los casos fue materializado con alcance social.

\section{Discusión}

Los trabajos de los estudiantes representan un alineamiento con la esencia de lo expresado por los expertos referenciados en el estado del arte, principalmente cuando el producto ha logrado integrar las competencias, el proyecto ético de vida y los procesos de emprendimiento creativo, siempre apuntando a la resolución de problemas de contexto de una manera sostenible. Es decir, se constituyen en conjuntos de acciones interrelacionadas claramente materializables para el logro de los objetivos planteados, que se evidencia en el énfasis de lo aprendido, en la relación que se establece con las disciplinas y, primordialmente, en que la investigación aplicada basada en el método científico es una aliada incondicional de la extensión en la óptica emprendedora.

La integración de conocimientos a través de proyectos resulta así una estrategia pedagógica práctica, dado que los intereses y esfuerzos del estudiante se concentran en un mismo producto, aprovechando de la mejor manera los recursos existentes, tales como el tiempo del trabajo independiente, los docentes del programa y demás recursos que facilita la universidad.

No hay duda de que en lo presentado en los trabajos "Implementing and operationalising integrative approaches to sustainability in higer education: the role of Project-oriented learning"[13], y "Between theory and practice: the importance of ICT in Higer Education as a tool for collaborative learning"[14], hay una clara alineación con lo realizado por los estudiantes de Tecnología Industrial de la Universidad de Santander, puesto que la aplicabilidad de los temas de desarrollo sostenible y la estrategia de aprendizaje por proyectos enfatizan la importancia del proyecto integrador como una herramienta de gran utilidad en la adquisición de las competencias requeridas a cualquier nivel, incluso hasta el de doctorado.

Es importante involucrar al estudiante en cada una de las fases propuestas, a fin de aumentar la motivación que permita a dicho estudiante ser protagonista en el proyecto integrador. 
La experiencia latinoamericana también representa un flujo de prácticas importantes dentro de la combinación de esfuerzos de estudiantes y docentes, así como de recursos de las universidades. Esto alineado con las competencias de formación esperadas como egresados, y que se validan en la utilidad que presentan con el carácter social con el cual se materializan los resultados de los proyectos realizados durante todo el proceso formativo que desembocan en trabajos de grado y/o en productos útiles a comunidades vulnerables.

Al observar comparativamente las competencias logradas por los estudiantes en los respectivos espacios mencionados en el estado del arte, si se recurre al caso de la Universidad de Sonora, en México, las competencias adquiridas por los estudiantes se centran en contenidos matemáticos para estudiar y aprender con didácticas que utilizan de manera inmediata en la resolución de problemas reales, desarrollando diversas habilidades y destrezas, tanto técnicas, como sociales, con los que se pueden enfrentar a diversas situaciones. Por su parte, los estudiantes de la Universidad de Santander hacen aprehensión de manera integral de los conocimientos, tanto de ciencias exactas, como sociales, que, como valor agregado, combinan con sus experiencias de trabajo, y así proponen soluciones a problemas del entorno social y empresarial. En este sentido, si la comparación se aborda con la Universidad de Málaga en España, las 10 capacidades mencionadas allí son el hilo conductor del estudiante de tecnología de la UDES, que mediante los seis semestres de formación desarrolla de manera disciplinar y electiva a fin de producir el impacto de su trabajo madurado durante este tiempo de formación. No solo para hacer visible el esfuerzo en encuentros de semilleros de investigación, sino más allá en su trabajo de grado y su proyecto de vida. Las cohortes de estudiantes evidencian que de 40 estudiantes que inician el ciclo formativo, hay una culminación satisfactoria del $70 \%$; los estudiantes rezagados aplazan, pero finalmente cumplen con el ciclo formativo apoyándose en el proyecto integrador.

Lo expresado en el párrafo anterior se alinea con la experiencia de la Universidad Autónoma de Colombia, que enfatiza de manera importante en la comprensión del estudiante de las técnicas de diseño e investigación significativas con proyectos destacados por su contenido social, el grado de interdisciplinariedad y la aplicación rigurosa de elementos metodológicos; asegura también que siempre hay detrás de cada proyecto un proceso intenso de indagación, de interacción, de compenetración con problemas reales de la comunidad por parte del estudiante. Nótese el énfasis tanto de la Autónoma, como de la UDEs, en el abordaje de los temas sociales como un reto para que los estudiantes estén a la vanguardia de proponer las soluciones y enfrentar las situaciones reales de un mundo cada vez mas complejo.

\section{Conclusiones}

El docente juega un papel fundamental en el desarrollo del proyecto integrador, dada la orientación que debe generar al estudiante para garantizar el cumplimiento de objetivos propuestos en dicho proyecto integrador, de tal manera que al existir una articulación directa y real entre la academia y las necesidades sociales, se puede asegurar una mejor apropiación del conocimiento y el desarrollo de competencias. Esto, al generar soluciones a problemas existentes, lo que haría posible que el proyecto integrador brinde la oportunidad a los estudiantes de generar y materializar una idea de negocio, siendo una alternativa que promueve la innovación mediante la creación de unidades productivas que, a largo plazo, se pueden convertir en una fuente de estabilidad económica. Es decir, el diseño de estrategias pedagógicas en el aula de clase contribuye a lograr un mayor acercamiento a la problemáticas del entorno que requieren soluciones alternativas propuestas desde la creatividad de los estudiantes y orientadas por el docente, a fin de integrar los conocimientos que se apropian para su aplicación. Por su parte, el docente debe tener claridad de la dinámica del proyecto integrador para poderlo difundir y garantizar el logro de los objetivos. Asimismo, es importante que se apropie de la metodología, mostrándose como un líder entre sus estudiantes y pueda así guiar correctamente al equipo de trabajo. Es de suma importancia mantener la motivación desde la planeación clara del desarrollo del proyecto, con tiempos reales de aplicación, ajustándose al calendario de actividades de la institución de tal manera que garantice el buen resultado de las actividades planteadas y culminar el trabajo satisfactoriamente. 


\section{Referencias}

[1] H. Etzkowitz, The Triple helix:academy-industry-government innovation in action, Routledge, 2008. Disponible en: https://books.google.com.co/books?id= hs2SAgAAQBAJ\&printsec $=$ frontcover \&dq=inauthor:\%22Henry+Etzkowitz\%22\&hl=es\&sa=X\&ve$\mathrm{d}=0$ ahUKEwjzkYSmxjTAhUK4CYKHZvHCkwQ6AEIJDAA\# $\mathrm{v}=$ onepage \&q\& $\mathrm{f}=$ false

[2] I. Fernandez, E. Castro, F. Conesa y A. Gutierrez, "Las relaciones universidad-empresa: entre la transferencia de resultados y el aprendizaje regional", Revista Espacios, vol. 21, 2000. Disponible en: http://www. revistaespacios.com/a00v21n02/60002102.html

[3] Planeación udes, Plan de Desarrollo de la Universidad de Santander 2013-2018, Bucaramanga, Colombia, 2013. Dispoinble en: http://www.udes.edu.co/ images/ la_universidad/documentos/Plan-de-Desarrollo_2013-2018.pdf

[4] UDEs, Proyecto Educativo Instituciona, Bucaramanga, Colombia, 2014. Disponible en: http://www. udes.edu.co/images/la_universidad/documentos/ PEI_MARZO_21_DE_2014.pdf

[5] S. Tobón, "Conferencia: El modelo de competencias en las prácticas docentes: Hacia escenarios significativos de vida", Revista Suma por la Educación, vol. 1, pp. 1-3, 2010.

[6] N. M. López-Rodríguez, El proyecto Integrador: Estrategia didáctica para la formación de competencias desde la perspectiva del enfoque socioformativo, $1^{\mathrm{a}}$ edición, México: Gafra Editores, 2012. Disponible en: http:// www.cinefop.com/home/index.php? option $=\mathrm{com}_{-}$ content\&view $=$ article\&id $=8 \&$ Itemid $=108$

[7] S. Tobón, Formación integral y competencias. Pensamiento complejo, currículo, didáctica y evaluación, $4^{\mathrm{a}}$ edición, Publicaciónes Ecoe Bogotá D. C., 2010. Disponible en: https://es.slideshare.net/mic401/libro-formacionintegralycompetencias-tobn-4ta-ed-40456182

[8] S. Tobón, J. Pimiento y J. García, Secuencias didácticas: aprendizaje y evaluación de competencias, 1a edición, México, Editorial Pearson, 2010. Disponible en: https://es.slideshare.net/anedortiz/libro-secuencias-didcticas-de

[9] H. Suárez, H. Soler y N. Gutiérrez, "Los Proyectos Integrados de semestre como estrategia de aprendizaje basado en proyectos, en el Programa de Ingeniería Electromecánica de la Fundación Universidad Autónoma de Colombia", Encuentro Internacional de Educación en Ingeniería, Cartagena, Colombia, Acofipaper, pp. 3-6. Disponible en:https://www.acofipapers. org/index.php/ei/2014/paper/viewFile/700/256

[10] A. García-Olalla y M. Poblete Ruiz, Una experiencia de aprendizaje cooperativo entre profesorado en el desarrollo de un proyecto de innovación docente, Páginas personales de la Universidad de Deusto, 2007.

[11] J. I. Gómez-Cordonié, Programación didáctica del proyecto integrado Creando empresa, Revista I.E.S., n. ${ }^{\circ} 1$, pp. 3- 14, 2015.

[12] J. L. Díaz-Gómez y P. D. López-Ceballos, "Proyectos, Integrados de Aprendizaje para Matemáticas. Nivel Medio Superior y Superior", en Memorias XV Semanas de la Universidad de Sonora, pp. 33-38, 2005. Disponible en: https://www.academia.edu/ 1050528/Proyectos_Integrados_de_Aprendizaje_ para_Matem\%C3\%A1ticas?

[13] 13] W. Filho Leal, C. Shiel y A. Paco, "Implementing and operationalising integrative approaches to sustainability in higer education: the role of Project-oriented learning", Journal of Cleaner Production, vol. 133, pp. 126-135. Disponible en: www.sciencedirect. com/science/article/pii/S0959652616305431

[14] N. Duta y O. Martínez-Rivera, "Between theory and practice: the importance of ICT in Higer Education as a tool for collaborative learning", Procedia-Social and Behavioral Sciences, vol. 180, pp. 1466-1473, 2014. doi: https://doi.org/10.1016/j.sbspro.2015.02.294

[15] D. M. Parra-Pineda, Manual de estrategias de enseñanza/aprendizaje, sENA, Regional Antioquia, la edición. Disponible en: http://epo86neza.com/comunicados/comunicado11.pdf

[16] C. Revelo y V. Rivas, "El proyecto integrador como investigación en el aula," I Congreso Latinoamericano de Enseñanza en Diseño, Universidad de Palermo, 2008. Disponible en: http://www.palermo.edu/dyc/ congreso-latino/pdf/Revelo.pdf

[17] Cámara de Comercio de Bogotá, El docente como promotor y formador de emprendimiento-guía básica, Cámara de Comercio de Bogotá, 2009. Disponible en: http://www.empresario.com.co/recursos/be/portal ninos/contenido/docleldocentecomopromotoryfor madordelemprendimiento.pdf

[18] H. A. Castillo, "Estado del arte en la enseñanza del emprendimiento", INTEC-Chile, 1999. Disponible en: http://recursos.ccb.org.co/bogotaemprende/portalninos/contenido/doc2estadodelarteenlaensenanzadele mprendimiento.pdf

[19] Universitaria Virtual Internacional, Metodología para desarrollar una actividad académica en entornos virtuales de aprendizaje, Bogotá D. C., Universitaria Virtual Internacional, 2013. Disponible en: http://www. uvirtual.edu.co/ Documents/Documentos-institucionales/Metodo-logia-Desarrollar-Actividad-Academica-Entornos-Virtuales-Aprendizaje.pdf

[20] E. Urbina-Miranda, La Industria automotriz en Sonora: un análisis bajo el enfoque del sistema regional de innovación, Universidad de Sonora México, 2005. 
Disponible en: http://tesis.uson.mx/digital/tesis/ docs/19264/Introducci\%C3\%B3n.pdf

[21] OECD, A Guiding Framework for Entrepreneurial Universities. European Comission y OECD, 2012. Disponible en: https://www.oecd.org/site/cfecpr/ EC-OECD\%20Entrepreneurial\%20Universities\%20 Framework.pdf

[22] T. Gómez, HeInnovate ¿Cómo medir el nivel de Emprendimiento de una Universidad?, INAECU. 2014. Disponible en: http://www.inaecu.com/heinnovate-como-de-emprendedora-es-una-universidad/

[23] R. L. Amaya, M. P., Díaz, M. Y. García y M. M. Zapata, Ekoentapes un Enchape a Base de Materiales Reciclados para el Revestimiento de Muros en el Área de Acabados del sector de la Construcción, Trabajo de Grado, Universidad de Santander uDES, Bucaramanga, 2013. Disponible en: http://www. udes.edu.co/programas-tecnologicos/gestionindustrial/715-proyecto-integrador.html

[24] R. J. Hurtado, B. R. Vilá y B. S. Vanesa, "La investigación formativa como metodolgía de aprendizaje en la mejora de competencia transversales", Procedia-Social and Behavioral Sciences, vol. 196, pp. 117-182, 2014. Disponible en: http://www.sciencedirect.com/ science/article/pii/S1877042815040239

[25] J. Roca, M. Reguant y O. Canet, (2015). Aprendizaje basado en problemas, estudio de casos y metodología tradicional: una experiencia concreta en el grado en enfermería. Procedia - Social and Behavioral Sciences. vol. 196, pp. 163-170. Disponible en: https://www.
researchgate.net/publication/282556329_Aprendizaje_Basado_en_Problemas_Estudio_de_Casos_y_ Metodologia_Tradicional_Una_Experiencia_Concreta_en_el_Grado_en_Enfermeria-

[26] C. J. Severiche y F. C. Pacheco, Plan de negocios para la producción y comercialización de ladrillos ecológicos-ecoladrillo, Trabajo de grado, Universidad de Santander UDES, Bucaramanga, 2015. Disponible en: http://www.udes.edu.co/images/programas/tec_gestion_industrial/plan_de_negicos_para_la_produccion_de_ladrillos.pdf

[27] B. C. Ortega, "Tecnologías de la información y la comunicación para la innovación educativa", Perfiles Educativos del Instituto de Investigaciones de la Universidad y la Educación, vol. xxxvi, n. ${ }^{\circ} 144$, pp. 214-218, 2014. Disponible en: http://www.redalyc. org/pdf/132/13230751014.pdf

[28] P. M. Martín, G. E. Díaz y D. Barrio, "Metodología docente y evaluación por competencias: una experiencia en la materia Dirección de Producción", Investigaciones Europeas de Dirección y Economía de la Empresa, n. ${ }^{\circ} 18$, pp. 237-247, 2012. Disponible en: http://www.redalyc.org/ html/2741/274124906007/

[29] C. L. Urbina, "Donarán utensilios amigables con el medio ambiente en Bucaramanga", Vanguardia.com, 22 mayo 2015. Disponible en: http://m.vanguardia. com/area-metropolitana/bucaramanga/312554-donaran-utensilios-amigables-con-el-medio-ambiente-en-bucarama 